\title{
Hierarchically Porous and Water-Tolerant Metal-Organic Frameworks for Enzyme Encapsulation
}

Yiying Sun, ,a,c, Jiafu Shi, ,b,c * Shaohua Zhang, ,a,c Yizhou Wu, a,c Shuang Mei, a,c Weilun Qian, ${ }^{a}$ Zhongyi Jianga,c

${ }^{a}$ Key Laboratory for Green Chemical Technology of Ministry of Education, School of Chemical Engineering and Technology, Tianjin University, Tianjin 300072, P. R. China.

${ }^{b}$ Tianjin Key Lab of Biomass/Wastes Utilization, School of Environmental Science \& Engineering, Tianjin University, Tianjin 300072, P. R. China.

${ }^{c}$ Collaborative Innovation Center of Chemical Science and Engineering (Tianjin), Tianjin 300072, P. R. China.

${ }^{*}$ Corresponding author: Jiafu Shi (shijiafu@tju.edu.cn)

KEYWORDS. Enzyme Encapsulation; Metal-Organic Frameworks;

Hierarchically Porous Structure; Water-Tolerant Property; Enhanced Activity and Stabilities 

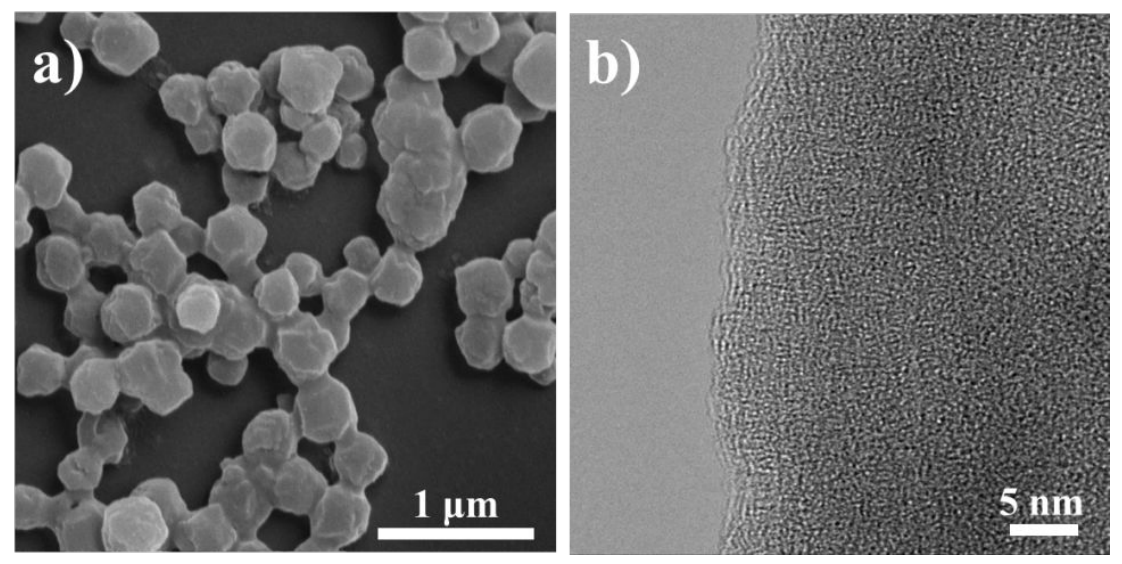

Figure S1. (a) SEM images of ZIF-8. (b) TEM images of partial enlargement of the edge ZIF-8@ZnTA. Note: ZIF-8@ZnTA was treated by TA solution (2.5mg mL-1, $\mathrm{pH} 7.0)$. 

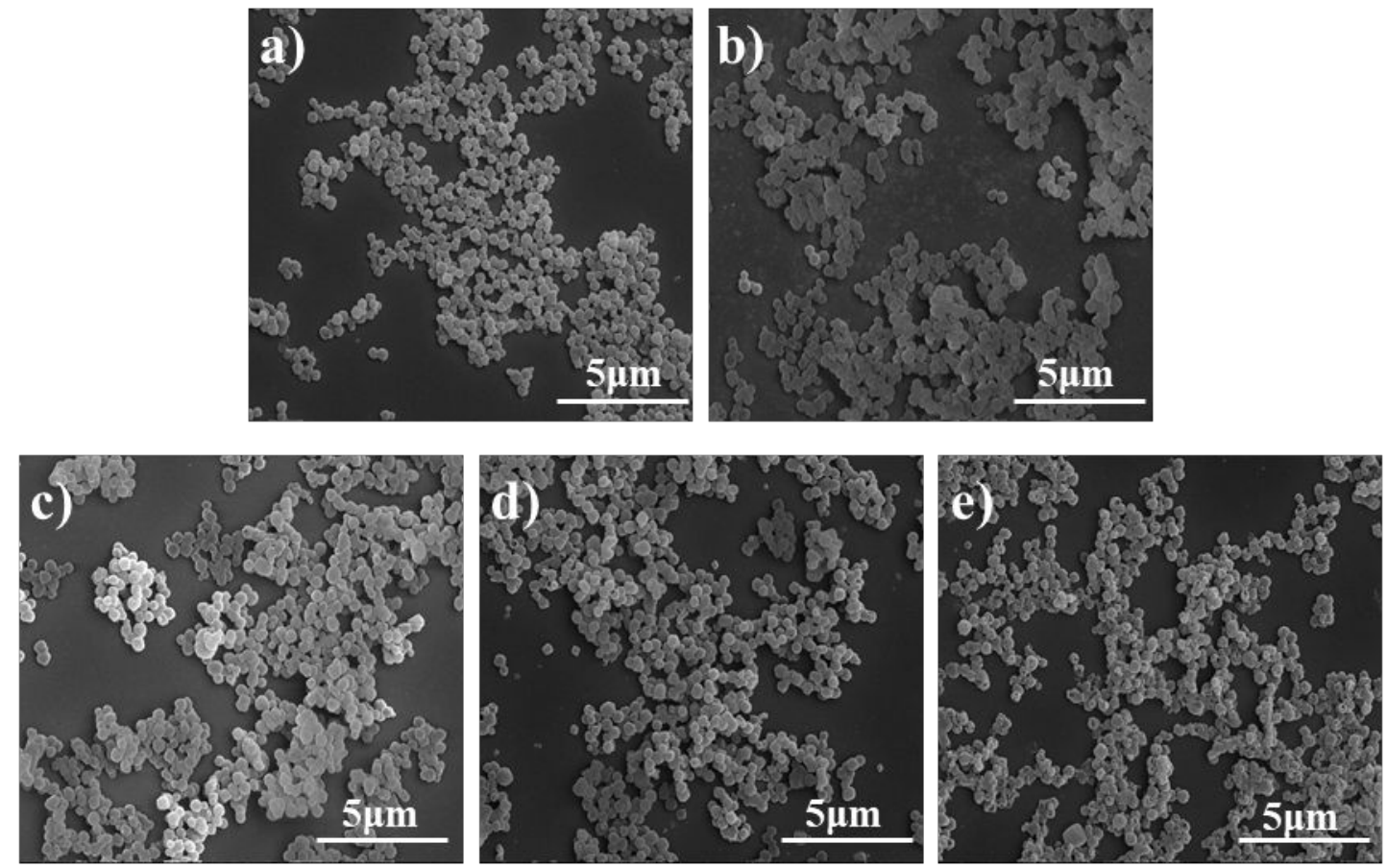

Figure S2. Large range SEM images of (a) ZIF-8, (b) 3.6 ZIF-8@ZnTA, (c) 5.5 ZIF-8@ZnTA, (d)7.0ZIF-8@ZnTA and (e)8.5 ZIF-8@ZnTA. 

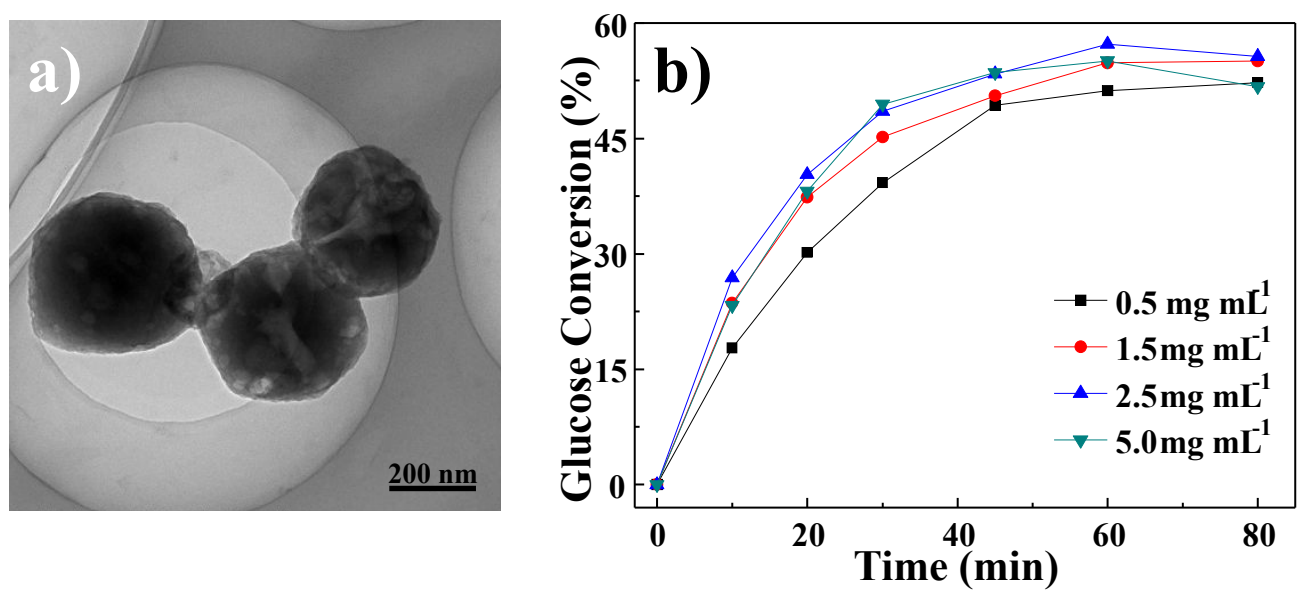

Figure S3. (a) TEM image of GOD@ZIF-8@ZnTA prepared under TA concentration of $0.25 \mathrm{mg} \mathrm{mL}$ at $\mathrm{pH}$ 7.0. (b) Glucose conversion catalyzed by GOD@ZIF-8@ZnTA prepared under different TA concentrations at pH 7.0.

The GOD@ZIF-8@ZnTA biocatalysts prepared under different TA concentrations at $\mathrm{pH} 7.0$ were then applied to catalyze the conversion of $\beta$-D-glucose. With the increase of TA concentration, the GOD@ZIF-8@ZnTA exhibited continuously increased $\beta$-D-glucose conversion ratio, and the lowest activity of GOD@ZIF-8@ZnTA prepared under TA concentration of $0.25 \mathrm{mg} \mathrm{mL}^{-1}$ at pH 7.0 should be owing to the rare-existence of macropores inside that may reduce the diffusion rate of $\beta$-D-glucose. 

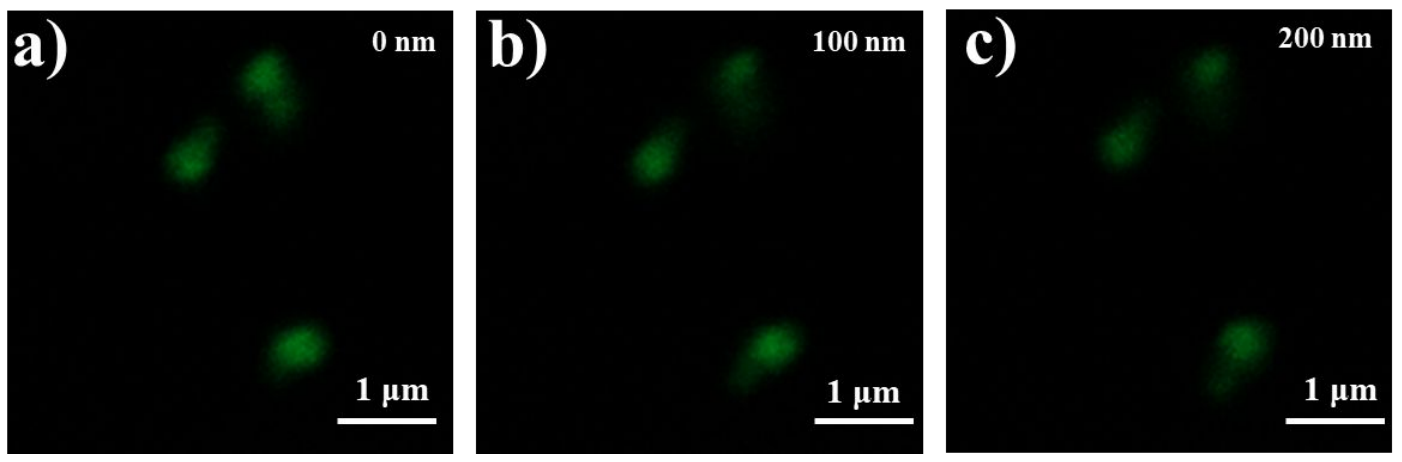

Figure S4. CLSM images of GOD@ZIF-8@ZnTA at different depths: (a) 0 nm, (b) $100 \mathrm{~nm}$, (c) $200 \mathrm{~nm}$. Note: GOD was labeled with fluorescin isothiocyanate (FITC).

As shown in Figure S4, the fluorescent light was detected at different depths of GOD@ZIF-8@ZnTA particles, which suggested that GOD was mainly located inside ZIF-8@ZnTA. 

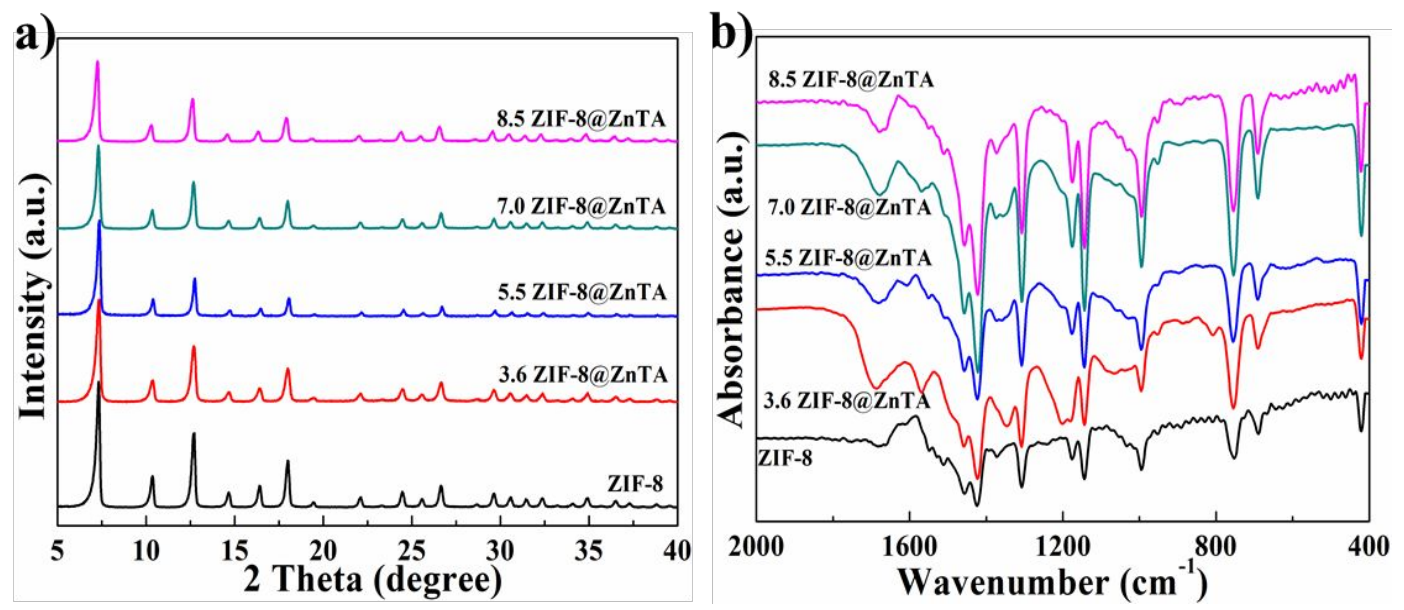

Figure S5. (a) XRD patterns and (b) FTIR spectra of ZIF-8@ZnTA treated by TA at different $\mathrm{pH}$ values $(\mathrm{pH} 3.6, \mathrm{pH}$ 5.5, $\mathrm{pH} 7.0, \mathrm{pH} 8.5)$ and ZIF-8.

As shown in Figure S5, the crystalline structure of ZIF-8@ZnTA with different pH values seemed to be unaltered when compared with solid ZIF-8, indicating the intrinsic framework of ZIF-8 retained during TA etching process with different $\mathrm{pH}$ values. Such ZIF-8 portion structure retention in ZIF-8@ZnTA with different pH values was also confirmed by the FTIR results. 

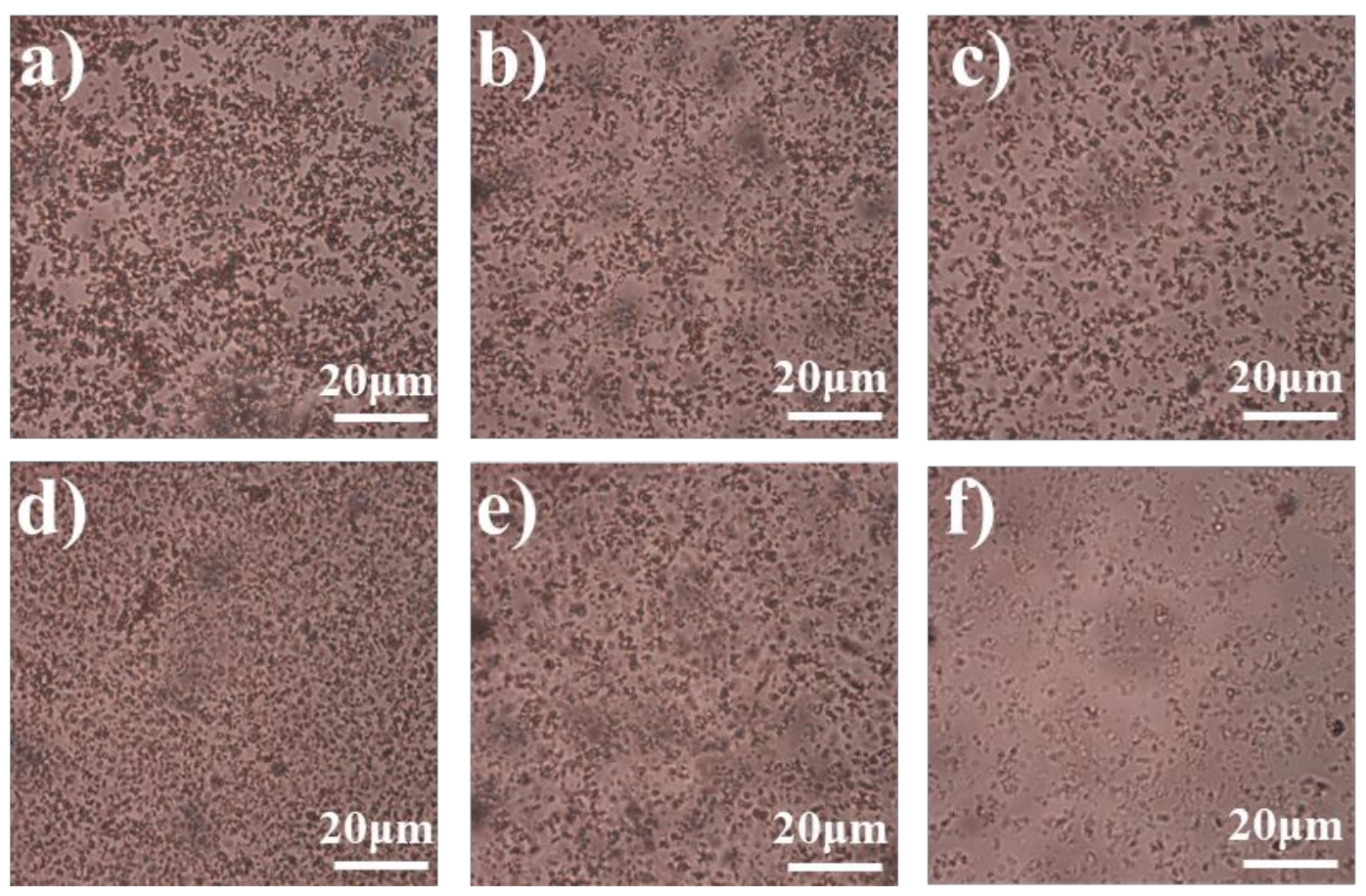

Figure S6. OM images of (a) GOD@ZIF-8@ZnTA, (b) GOD@ZIF-8@ZnTA after cycling tests (C-GOD@ZIF-8@ZnTA), (c) GOD@ZIF-8@ZnTA after thermal tests (T-GOD@ZIF-8@ZnTA), (d) GOD@ZIF-8, (e) GOD@ZIF-8 after cycling tests (C-GOD@ZIF-8) and (f) GOD@ZIF-8 after thermal tests (T-GOD@ZIF-8).

After the thermal and cycling stability tests, the suspensions of GOD@ZIF-8 and GOD@ZIF-8@ZnTA were collected for optical microscope measurement. As shown in the OM images, 76.4\% (2752/3404) of GOD@ZIF-8@ZnTA particles were preserved (Figure S6a and S6b) after 4 times of cycling (10 min for 1 cycling) while only 46.3\% (2219/5230) left for GOD@ZIF-8 particles (Figure S6d and S6e). After heated at $70{ }^{\circ} \mathrm{C}$ for 2 hours, 50.3\%(1711/3404) of GOD@ZIF-8@ZnTA particles were preserved (Figure S6a and S6c) while only 17.6\% (921/5230) left for GOD@ZIF-8 particles (Figure S6d and S6f). The high retention of intact particles of GOD@ZIF-8@ZnTA contributed a lot to the better remained activity of GOD@ZIF-8@ZnTA. 


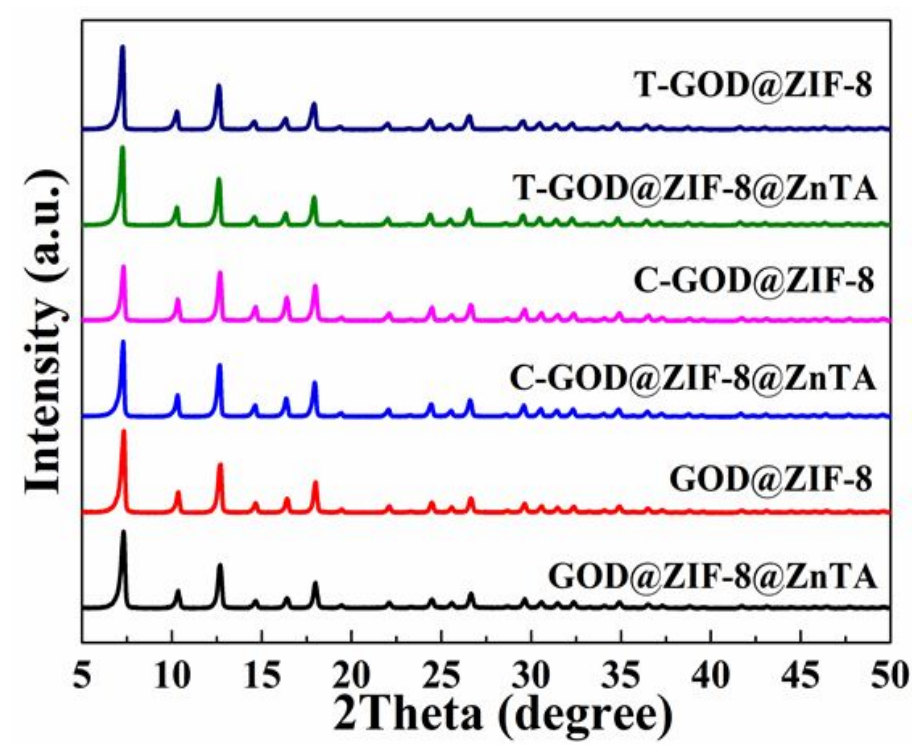

Figure S7. XRD patterns of GOD@ZIF-8, GOD@ZIF-8@ZnTA, T-GOD@ZIF-8, T-GOD@ZIF-8@ZnTA, C-GOD@ZIF-8 and C-GOD@ZIF-8@ZnTA.

The sedimentation was collected for XRD characterization after centrifugation. As shown in Figure S7, the crystalline structure of GOD@ZIF-8@ZnTA and GOD@ZIF-8 seemed to be unaltered after the thermal $\left(70{ }^{\circ} \mathrm{C}\right)$ and cycling tests $(4$ times), which suggested the intrinsic structure of ZIF-8 was remained during the stability tests. 


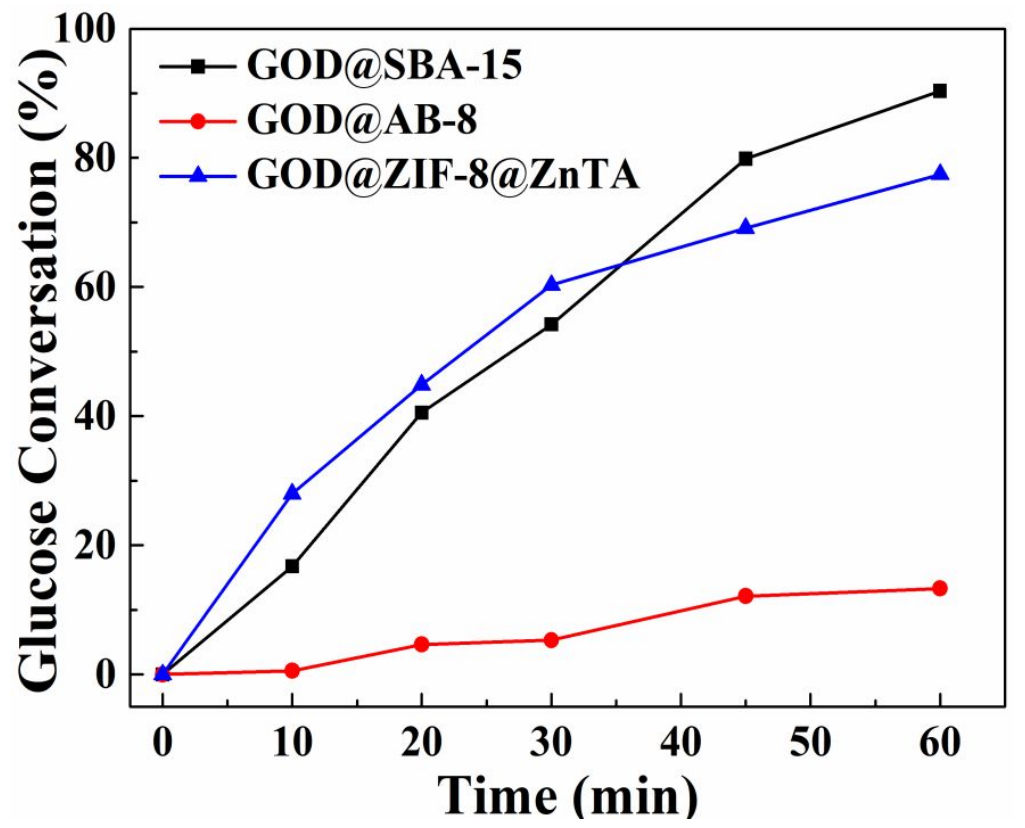

Figure S8. $\beta$-D-glucose conversion with reaction time catalyzed by the GOD encapsulated ZIF-8@ZnTA (GOD@ZIF-8@ZnTA), GOD absorbed in mesoporous silica SBA-15 (GOD@SBA-15), and GOD absorbed in macroporous resin AB-8 (GOD@AB-8). Note: All samples were tested with equal mass amount of catalysts (1.5 mg).

As examined in our study, GOD@ZIF-8@ZnTA exhibited an enzyme immobilization efficiency of 80\%, much higher than GOD@SBA-15 (58\%) and GOD@AB-8 (52\%). When applied to catalyze the oxidation of glucose in the presence of oxygen, GOD@ZIF-8@ZnTA exhibited an equilibrium glucose conversion ratio of 78\%, a bit lower than GOD@SBA-15 (89\%) but still much higher than GOD@AB-8 (14\%). However, the leakage ratios of GOD@SBA-15 and GOD@AB-8 were, respectively, 22\% and 25\%. By contrast, there was almost no enzyme leaching for GOD@ZIF-8@ZnTA $(<1.2 \%)$. It should be further noted that the higher catalytic rate of GOD@SBA-15 after 30 min might be caused by the leaching of free GOD (Figure S8). Moreover, the much lower activity of GOD@AB-8 might be due to the insufficient contact of substrate (glucose and oxygen) and immobilized GOD, which was the inherent drawbacks of millimeter-sized AB-8 when compared with nanoscale MOFs or SBA-15. 
Table S1. Enzyme immobilization efficiency and leakage ratio of GOD@ZIF-8@ZnTA,GOD@SBA-15 and GOD@AB-8.

\begin{tabular}{c|c|c|c}
\hline & GOD@ZIF-8@ZnTA & GOD@SBA-15 & GOD@AB-8 \\
\hline $\begin{array}{c}\text { Enzyme immobilization } \\
\text { efficiency } / \%\end{array}$ & 80.6 & 58.3 & 51.9 \\
\hline $\begin{array}{c}\text { Enzyme leakage ratio } \\
/ \%\end{array}$ & 1.2 & 21.9 & 25.0 \\
\hline
\end{tabular}

